TRANSACTIONS OF THE

AMERICAN MATHEMATICAL SOCIETY

Volume 356, Number 5, Pages 1875-1888

S 0002-9947(03)03421-4

Article electronically published on October 8, 2003

\title{
UNIQUENESS OF VARIETIES OF MINIMAL DEGREE CONTAINING A GIVEN SCHEME
}

\author{
M. CASANELLAS
}

\begin{abstract}
We prove that if $X \subset \mathbb{P}^{N}$ has dimension $k$ and it is $r$-Buchsbaum with $r>\max (\operatorname{codim} X-k, 0)$, then $X$ is contained in at most one variety of minimal degree and dimension $k+1$.
\end{abstract}

\section{INTRODUCTION}

The purpose of this paper is to compute how many varieties of minimal degree and dimension $k+1$ contain a given scheme $X \subset \mathbb{P}^{N}$ of dimension $k \geq 1$. If $X$ is arithmetically Buchsbaum, then there might be more than one variety of minimal degree containing it, but in this paper we prove that if $X$ is $r$-Buchsbaum with $r>\max (\operatorname{codim} X-k, 0)$, then $X$ is contained in at most one variety of minimal degree and dimension $k+1$ (see Corollary 4.10 and Theorem 4.9). In this case, the only varieties of minimal degree that can contain $X$ are rational normal scrolls, so this paper is mostly devoted to the study of divisors on rational normal scrolls. We would like to point out that the bound $r>\max (\operatorname{codim} X-k, 0)$ cannot be improved in general (see Remark 4.11).

To address this problem we will study the structure of the deficiency modules of $X$. As J. Migliore introduced them in [10], the structure of the deficiency modules can be studied by looking at certain varieties in $\left(\mathbb{P}^{N}\right)^{*}$. We will prove that the structure of the deficiency modules of a divisor on a rational normal scroll is strongly determined by the rational normal scroll containing it.

J. Migliore used the study of the structure of deficiency modules to classify unions of linear varieties in terms of liaison theory (see [10 and 11]). In this paper, we also use liaison theory as a tool and we also give consequences of our results in terms of liaison.

Now we outline the structure of the paper. In the following section we introduce the notation and the preliminaries on the structure of deficiency modules that we will use later. Section 3 is devoted to the study of the structure of deficiency modules of non-connected schemes and contains useful results on this subject. The last section contains the main results of this paper.

The results in this paper are part of my Ph.D. thesis [3]. I would like to thank my advisor, Prof. R.M. Miró-Roig, for checking these results so carefully and for all her fruitful advice, and I'd like to acknowledge Prof. E. Casas-Alvero for suggesting this problem to me.

Received by the editors August 5, 2002.

2000 Mathematics Subject Classification. Primary 14M06, 14M12, 14 M05.

(C)2003 American Mathematical Society 


\section{NotATION AND PRELIMINARIES}

Throughout this paper, $\mathbb{P}^{N}$ will be the $N$-dimensional projective space over an algebraically closed field $K$ of characteristic zero, and we will denote by $R$ the ring $R=K\left[X_{0}, \ldots, X_{N}\right]$. By a subscheme $V \subset \mathbb{P}^{N}$ we mean an equidimensional closed subscheme. If $V \subset \mathbb{P}^{N}$ is a subscheme, we denote by $\mathcal{I}_{V}$ its ideal sheaf and by $I(V)$ its saturated homogeneous ideal; note that $I(V)=\bigoplus_{t \in \mathbb{Z}} H^{0}\left(\mathbb{P}^{N}, \mathcal{I}_{V}(t)\right)$.

Most of the background needed in this paper can be found in the book of $\mathrm{J}$. Migliore 12. In order to make this paper more self-contained, we recall some of this background here.

Given a closed subscheme $V \subset \mathbb{P}^{N}$ of dimension $d \geq 1$, we define its deficiency modules as

$$
M^{i}(V)=\bigoplus_{t \in \mathbb{Z}} H^{i}\left(\mathbb{P}^{N}, \mathcal{I}_{V}(t)\right)
$$

for $1 \leq i \leq d$. The first deficiency module $M^{1}(V)$ is also called the Rao module (or Hartshorne-Rao module). As we will see, these modules give us a lot of geometric information about the scheme. We recall that a scheme of dimension $\geq 1$ is locally Cohen-Macaulay if and only if all of its deficiency modules have finite length (see 12], Theorem 1.2.5). From now on, all the schemes we consider will be locally Cohen-Macaulay. Any locally Cohen-Macaulay scheme is $r$-Buchsbaum for some $r \geq 0$. As the definition of $r$-Buchsbaum is somewhat inconsistent in the literature, we adopt the definition given in [14]:

Definition 2.1. Let $X \subset \mathbb{P}^{N}$ be a locally Cohen-Macaulay subscheme of dimension $\geq 1$. We say that $X$ is $r$-Buchsbaum if $r$ is the least integer such that $\mathfrak{m}^{r}$ annihilates all the deficiency modules $M^{i}(X), i=1, \ldots, \operatorname{dim} X$. We will also say that $r$ is the Ellia-Migliore-MiróRoig number of $X$.

Notice that arithmetically Cohen-Macaulay schemes (briefly ACM) and 0-Buchsbaum schemes coincide, and any arithmetically Buchsbaum scheme not arithmetically Cohen-Macaulay is 1-Buchsbaum. For curves, the converse also holds: a curve $C \subset \mathbb{P}^{n}$ is 1-Buchsbaum if and only if it is arithmetically Buchsbaum but not ACM; but this is no longer true in higher dimension (see for instance [13, Example 7.14). The reader can look at the paper of L.T. Hoa, R.M. Miró-Roig and W. Vogel [7], where a definition concerning the preservation of the $r$-Buchsbaum property under hyperplane sections is given.

In order to study the module structure of the deficiency modules of a subscheme $X \subset \mathbb{P}^{N}$ of dimension $k \geq 1$, we shall consider the following invariants introduced in [10]:

For any linear form $L \in R_{1}$, any $n \in \mathbb{Z}$ and any $i=1, \ldots, k$, we consider the homomorphism of K-vector spaces induced by $\mathcal{I}_{X}(n) \stackrel{\times L}{\rightarrow} \mathcal{I}_{X}(n+1)$ on the deficiency modules of $X$,

$$
\phi_{n}^{i}(L): M^{i}(X)_{n} \longrightarrow M^{i}(X)_{n+1} .
$$

The collection of homomorphisms

$$
\begin{aligned}
\phi_{n}^{i}: & R_{1} \longrightarrow \operatorname{Hom}_{K}\left(M^{i}(X)_{n}, M^{i}(X)_{n+1}\right), \\
& L \longmapsto \phi_{n}^{i}(L),
\end{aligned}
$$

$n \in \mathbb{Z}$, determines the module structure of $M^{i}(X)$ (for $i=1, \ldots, k$ ). We will be mainly interested in $\phi_{n}^{1}(L)$ (resp. $\phi_{n}^{1}$ ), which we will denote simply as $\phi_{n}(L)$ (resp. $\left.\phi_{n}\right)$, and we will specify the scheme $X$ as $\phi_{n}^{X}(L)$ (resp. $\phi_{n}^{X}$ ) if there is danger of 
ambiguity. We can also consider the degeneracy loci of $\phi_{n}$ and we can identify them with subvarieties of the dual projective space $\left(\mathbb{P}^{N}\right)^{*}$. We define

$$
V_{n, j}:=\left\{L^{*} \in\left(\mathbb{P}^{N}\right)^{*} \mid \operatorname{rk} \phi_{n}(L) \leq j\right\} \subseteq\left(\mathbb{P}^{N}\right)^{*}
$$

for any $j \in \mathbb{Z}$. Observe that $V_{n, j} \subseteq V_{n, j+1}$. So, if we set $s_{n}:=\max \left\{j \mid V_{n, j} \varsubsetneqq\right.$ $\left.\left(\mathbb{P}^{N}\right)^{*}\right\}$, we can define $V_{n}:=V_{n, s_{n}}$ (or we will be more specific, referring to $V_{n, j}(X)$ and $V_{n}(X)$ when necessary).

Whereas the $\phi_{n}$ are not invariants up to isomorphism of $M^{1}(X)$, the loci $V_{n, j}$ (and hence $V_{n}$ ) are isomorphism invariants, and they are preserved under shift (reindexing) and dual $\left(M^{1}(X)^{\vee}\right.$ has degeneracy loci $\left.V_{n, j}^{\vee}=V_{-n-1, j}\right)$. These invariants were introduced and deeply studied in [10] (the reader can also see the book of J. Migliore [12, sections 1.1 and 5.5). In the rest of the paper we will see that these invariants are strongly related to the geometry of the scheme we are considering.

We fix some more notation here: for any linear form $L \in R_{1}$, we denote by $H_{L}$ the hyperplane defined by $L$. For any scheme $X \subset \mathbb{P}^{N}$ of dimension $k \geq 1$, let $U_{X}$ be the following open subset of $\left(\mathbb{P}^{N}\right)^{*}$ :

$$
U_{X}:=\left\{L^{*} \in\left(\mathbb{P}^{N}\right)^{*} \mid \operatorname{dim} H_{L} \cap X=k-1\right\},
$$

i.e., $U_{X}$ is the set of all $L^{*} \in\left(\mathbb{P}^{N}\right)^{*}$ such that $H_{L}$ does not contain any irreducible component of $X$.

If $Z$ is any closed subscheme of $\mathbb{P}^{N}$, we denote by $\langle Z\rangle$ the span of $Z$, i.e., the least linear subspace of $\mathbb{P}^{N}$ containing $Z$ as a subscheme.

For the definition of G-liaison (Gorenstein liaison), CI-liaison (complete intersection liaison), G-linkage and more information about liaison theory, see [12] and 8]. The result that relates deficiency modules and liaison theory is the following (see for instance [12], Theorem 5.3.1):

Theorem 2.2 (Hartshorne-Schenzel). Let $V_{1}, V_{2} \subset \mathbb{P}^{N}$ be two schemes of dimension $d \geq 1$ in the same even G-liaison class. Then $M^{i}\left(V_{1}\right) \cong M^{i}\left(V_{2}\right)(t)$ for all $i=1, \ldots, d$ and some $t \in \mathbb{Z}$.

In particular, by the Hartshorne-Schenzel Theorem [2.2, the invariants $V_{n, r}$ introduced above are invariants of a given even G-liaison class (in fact, they are also invariants of CI-liaison classes).

\section{The STRUCTURE OF DEFICIENCY MODUlES}

Using the notation introduced in section 2, we first state a generalized version of [10, Proposition 2.1:

Lemma 3.1. Let $X \subset \mathbb{P}^{N}$ be a subscheme and let $L \in R_{1}$ be a linear form such that $L^{*} \in U_{X}$. Then $L^{*} \in U_{X} \cap V_{n}$ if and only if there exists $M^{*} \in U_{X}$ such that

$$
h^{0}\left(H_{L}, \mathcal{I}_{X \cap H_{L}, H_{L}}(n+1)\right)>h^{0}\left(H_{M}, \mathcal{I}_{X \cap H_{M}, H_{M}}(n+1)\right) .
$$

Proof. Since $L^{*} \in U_{X}$, we have an exact sequence of sheaves

$$
0 \longrightarrow \mathcal{I}_{X}(n) \stackrel{\times L}{\longrightarrow} \mathcal{I}_{X}(n+1) \longrightarrow \mathcal{I}_{X \cap H_{L}, H_{L}}(n+1) \longrightarrow 0
$$

for all $n \in \mathbb{Z}$ and analogously for any $M^{*} \in U_{X}$. Taking cohomology in (1) and cutting it, we get

(2) $0 \rightarrow \operatorname{Coker}(\times L) \rightarrow H^{0}\left(\mathcal{I}_{X \cap H_{L}, H_{L}}(n+1)\right) \rightarrow M^{1}(X)_{n} \stackrel{\phi_{n}(L)}{\longrightarrow} M^{1}(X)_{n+1} \rightarrow \cdots$ 
and similarly for any $M^{*} \in U_{X}$. But $\operatorname{dim}_{K}$ Coker $(\times L)$ does not depend on the choice of $L$, i.e., $\operatorname{dim}_{K} \operatorname{Coker}(\times L)=\operatorname{dim}_{K} \operatorname{Coker}(\times M)$ for all $L^{*}, M^{*} \in$ $U_{X}$. Therefore, we deduce from (2) that $\operatorname{rk} \phi_{n}(L)<\operatorname{rk} \phi_{n}(M)$ if and only if $h^{0}\left(\mathcal{I}_{X \cap H_{L}, H_{L}}(n+1)\right)>h^{0}\left(\mathcal{I}_{X \cap H_{M}, H_{M}}(n+1)\right)$ (for $L^{*}$ and $M^{*}$ in $\left.U_{X}\right)$. Since $L^{*}$ belongs to $V_{n}$ if and only if $\operatorname{rk} \phi_{n}(L)<\operatorname{rk} \phi_{n}(M)$ for a general $M \in R_{1}$, the lemma is proved.

The following lemma tells us what happens when $L^{*}$ does not belong to $U_{X}$ as long as $X$ is a non-connected subscheme:

Lemma 3.2. Let $X=\bigcup_{i=1}^{r} Y_{i} \subset \mathbb{P}^{N}$ be the disjoint union of $r$ schemes of dimension $k \geq 1, r \geq 2$. Suppose that the general hyperplane section of $X$ is non-degenerate (in particular, $X$ is non-degenerate). If $H_{L}$ contains $Y_{i}$ for some $i=1, \ldots, r$, then $L^{*} \in V_{0}$.

Proof. We observe first that, since $X$ is a non-connected scheme, $M^{1}(X)_{0} \neq 0$ (see [12], Theorem 1.2.6). For a general $M \in R_{1}$ we have the exact sequence of sheaves

$$
0 \rightarrow \mathcal{I}_{X} \rightarrow \mathcal{I}_{X}(1) \rightarrow \mathcal{I}_{X \cap H_{M}, H_{M}}(1) \rightarrow 0
$$

that induces the exact sequence on cohomology

$$
0 \longrightarrow H^{0}\left(H_{M}, \mathcal{I}_{X \cap H_{M}, H_{M}}(1)\right) \longrightarrow M^{1}(X)_{0} \stackrel{\phi_{0}(M)}{\longrightarrow} M^{1}(X)_{1} \longrightarrow \ldots
$$

Since, by assumption, $X \cap H_{M}$ is non-degenerate, we obtain that $\operatorname{Ker} \phi_{0}(M)=0$, and since $M^{1}(X)_{0} \neq 0$, it makes sense to study $V_{0}$. In particular, for a general $M \in$ $R_{1}, \phi_{0}(M)$ is a vector space homomorphism which has rank equal to $\operatorname{dim}_{K} M^{1}(X)_{0}$.

Now we come to the study of $\operatorname{rk} \phi_{0}(L)$ when $H_{L}$ contains $Y_{i}$ for some $i$. We want to prove that $\phi_{0}(L)$ has a non-trivial kernel. Without loss of generality, we may assume that $H_{L} \supset Y_{1}$, and we write $X=Y_{1} \cup Z$ with $Z=\bigcup_{i=2}^{r} Y_{i}$. As usual, we have the exact sequence

$$
0 \longrightarrow I(X)=I\left(Y_{1}\right) \cap I(Z) \longrightarrow I\left(Y_{1}\right) \oplus I(Z) \longrightarrow I\left(Y_{1}\right)+I(Z) \longrightarrow 0 .
$$

Since $Y_{1} \cap Z=\emptyset$, we have $\mathcal{I}_{Y_{1}}+\mathcal{I}_{Z} \cong \mathcal{O}_{\mathbb{P}^{N}}$, so the exact sequence above induces (sheafifying and taking cohomology) the following exact sequence of $R$-modules:

$$
0 \longrightarrow R /\left(I\left(Y_{1}\right)+I(Z)\right) \longrightarrow M^{1}(X) \rightarrow M^{1}\left(Y_{1}\right) \oplus M^{1}(Z) \longrightarrow \ldots
$$

We can also consider the multiplication by $L$, so that this exact sequence (in degrees 0 and 1 ) leads to the following commutative diagram with exact rows:

$$
\begin{aligned}
& 0 \rightarrow \quad K \quad \rightarrow \quad M^{1}(X)_{0} \quad \rightarrow \quad M^{1}\left(Y_{1}\right)_{0} \oplus M^{1}(Z)_{0} \cdots \\
& \downarrow \times L \quad \downarrow \phi_{0}(L) \quad \downarrow \phi_{0}^{Y_{1}}(L) \oplus \phi_{0}^{Z}(L) \\
& 0 \rightarrow\left(R /\left(I\left(Y_{1}\right)+I(Z)\right)\right)_{1} \quad \rightarrow \quad M^{1}(X)_{1} \quad \rightarrow \quad M^{1}\left(Y_{1}\right)_{1} \oplus M^{1}(Z)_{1} \cdots
\end{aligned}
$$

In particular, by the snake lemma, we have an exact sequence

$$
0 \longrightarrow \operatorname{Ker}(\times L) \longrightarrow \operatorname{Ker}\left(\phi_{0}(L)\right) \longrightarrow \operatorname{Ker}\left(\phi_{0}^{Y_{1}}(L) \oplus \phi_{0}^{Z}(L)\right)
$$

As $H_{L} \supset Y_{1}, L$ belongs to $I\left(Y_{1}\right)_{1}$ and so $[L]=0$ in $\left(R /\left(I\left(Y_{1}\right)+I(Z)\right)\right)_{1}$. This means that $K \stackrel{\times L}{\longrightarrow}\left(R /\left(I\left(Y_{1}\right)+I(Z)\right)\right)_{1}$ is the zero map and $\operatorname{Ker}(\times L) \cong K$. But then from (3) we can deduce that $\operatorname{Ker} \phi_{0}(L) \neq 0$, and hence $\operatorname{rk} \phi_{0}(L)<\operatorname{rk} \phi_{0}(M)$ for a general $M$. Thus, $L^{*} \in V_{0}$, as we wanted to prove. 
If $X$ is a degenerate scheme, we have

Lemma 3.3 (12], Proposition 1.3.10 (b)). If $X \subset \mathbb{P}^{N}$ is a degenerate subscheme of dimension $k \geq 1$ and $H_{L}$ is a hyperplane containing $X$, then $\phi_{n}^{i}(L)=0$ for all $i=1, \ldots, k$ and $n \in \mathbb{Z}$.

Now we will see that for a non-connected reduced scheme $X \subset \mathbb{P}^{N}$ having more connected components than $\operatorname{codim} X-\operatorname{dim} X+1$, the hyperplanes $H_{L} \supset X$ are the only ones satisfying $\phi_{0}^{X}(L)=0$. Indeed, we have

Lemma 3.4. Let $X=\bigcup_{i=1}^{n} Y_{i} \subset \mathbb{P}^{N}$ be the disjoint union of $n \geq 2$ subschemes of dimension $k \geq 1$. Assume that each $Y_{i}$ is reduced and connected and that $n>$ $N-2 k+1=\operatorname{codim} X-\operatorname{dim} X+1$. If $X \nsubseteq H_{M}$, then $\phi_{0}^{X}(M) \neq 0$.

Proof. Let $H_{M}$ be a hyperplane not containing $X, M \in R_{1}$. We first consider the case $M^{*} \in U_{X}$. In this case we have the following exact sequence on cohomology:

$$
\begin{aligned}
0=H^{0}\left(\mathcal{I}_{X}\right) \rightarrow H^{0}\left(\mathcal{I}_{X}(1)\right) \rightarrow H^{0}\left(H_{M}, \mathcal{I}_{X \cap H_{M}}(1)\right) & \rightarrow M^{1}(X)_{0} \\
& \stackrel{\phi_{0}^{X}(M)}{\longrightarrow} M^{1}(X)_{1} \rightarrow \ldots
\end{aligned}
$$

Suppose that $\phi_{0}^{X}(M)=0$. Then we could shorten the above sequence and obtain a short exact sequence:

$$
0 \rightarrow H^{0}\left(\mathbb{P}^{N}, \mathcal{I}_{X}(1)\right) \longrightarrow H^{0}\left(H_{M}, \mathcal{I}_{X \cap H_{M}, H_{M}}(1)\right) \longrightarrow M^{1}(X)_{0} \longrightarrow 0 .
$$

As $X$ is reduced and has $n$ connected components, $\operatorname{dim} M^{1}(X)_{0}=n-1$ (see [12, Theorem 1.2.6), the short exact sequence above would imply $h^{0}\left(H_{M}, \mathcal{I}_{X \cap H_{M}, H_{M}}(1)\right)$ $\geq n-1$. On the other hand, since $X \cap H_{M}$ is the union of $n \geq 2$ disjoint subschemes of dimension $k-1$, the linear span $\left\langle X \cap H_{M}\right\rangle$ has dimension at least $2 k-1$ and so $h^{0}\left(H_{M}, \mathcal{I}_{X \cap H_{M}, H_{M}}(1)\right) \leq N-1-2 k+1$. But this leads to a contradiction with the assumption $n>N-2 k+1$.

Now suppose that $H_{M}$ contains one of the components of $X$, for instance $H_{M} \supset$ $Y_{j}$. Let us call $Z$ the union $\bigcup_{i \neq j} Y_{i}$. Then we have the exact sequences of sheaves

$$
\begin{gathered}
0 \longrightarrow \mathcal{I}_{X} \stackrel{\iota}{\longrightarrow} \mathcal{I}_{Z} \longrightarrow \mathcal{O}_{Y_{j}} \longrightarrow 0, \\
0 \longrightarrow \mathcal{I}_{Z} \stackrel{\times M}{\longrightarrow} \mathcal{I}_{X}(1) \longrightarrow \mathcal{I}_{X \cap H_{M}, H_{M}}(1) \longrightarrow 0,
\end{gathered}
$$

and the composition $(\times M) \circ \iota$ induces the map $\phi_{0}^{X}(M)$ in cohomology. Thus, taking cohomology on both exact sequences, we get

$$
\begin{gathered}
0=H^{0}\left(\mathcal{I}_{Z}\right) \longrightarrow H^{0}\left(\mathcal{O}_{Y_{j}}\right) \longrightarrow M^{1}(X)_{0} \stackrel{\varphi}{\longrightarrow} M^{1}(Z)_{0} \longrightarrow \cdots, \\
0=H^{0}\left(\mathcal{I}_{Z}\right) \rightarrow H^{0}\left(\mathcal{I}_{X}(1)\right) \rightarrow H^{0}\left(H_{M}, \mathcal{I}_{X \cap H_{M}}(1)\right) \rightarrow M^{1}(Z)_{0} \stackrel{\psi}{\rightarrow} M^{1}(X)_{1} \rightarrow \cdots .
\end{gathered}
$$

Since $Y_{i}$ is reduced for any $i$, we have $h^{0}\left(\mathcal{O}_{Y_{j}}\right)=1, \operatorname{dim}_{K} M^{1}(X)_{0}=n-1$ and $\operatorname{dim}_{K} M^{1}(Z)_{0}=n-2$. So $\varphi$ is a surjective morphism of $\mathrm{K}$-vector spaces (checking dimensions). Therefore, the morphism $\phi_{0}^{X}(M)=\psi \circ \varphi$ is zero if and only if $\psi=$ 0 . But if $\psi$ were the zero map, it would imply that $h^{0}\left(H_{M}, \mathcal{I}_{X \cap H_{M}, H_{M}}(1)\right)=$ $h^{0}\left(\mathcal{I}_{X}(1)\right)+\operatorname{dim}_{K} M^{1}(Z)_{0} \geq n-2$. Furthermore,

$$
X \cap H_{M}=Y_{j} \cap H_{M} \cup \bigcup_{i \neq j} Y_{i} \cap H_{M}
$$


spans a projective space of dimension at least $2 k$ (because $\operatorname{dim} Y_{j} \cap H_{M}=k$ and $n \geq 2)$. Thus $n-2 \leq h^{0}\left(H_{M}, \mathcal{I}_{X \cap H_{M}, H_{M}}(1)\right) \leq N-1-2 k$, which contradicts the assumption $n>N-2 k+1$. Hence, the lemma is proved.

Remark 3.5. It can be checked that Lemma 3.4 is still true if we change the assumption $n>N-2 k+1$ to $n>N-2 k+1-\varepsilon_{X}$, where $\varepsilon_{X}:=h^{0}\left(\mathbb{P}^{N}, \mathcal{I}_{X}(1)\right)$. However, the assumption $n>N-2 k+1-\varepsilon_{X}$ cannot be avoided: for instance, if we consider $X \subset \mathbb{P}^{3}$ to be the disjoint union of two lines, then $N=3, k=1$, $n=2, \varepsilon_{X}=0, X$ is an arithmetically Buchsbaum scheme, and $\phi_{0}^{X}(L)=0$ for all $L \in K\left[X_{0}, X_{1}, X_{2}, X_{3}\right]_{1}$.

Lemmas 3.3 and 3.4 suggest that if we consider two degenerate schemes with isomorphic deficiency modules, then there exists a close relation between the hyperplanes containing them. Indeed, when we restrict to the hypothesis of Lemma 3.4 we have

Proposition 3.6. Let $X=\bigcup_{i=1}^{n} Y_{i}$ and $X^{\prime}=\bigcup_{i=1}^{n^{\prime}} Y_{i}^{\prime}$ be the disjoint union of $n \geq 2$ (resp. $n^{\prime}$ ) connected and reduced schemes of dimension $k \geq 1$. Suppose that $X, X^{\prime}$ are subschemes of $\mathbb{P}^{N}$ and $n>N-2 k+1=\operatorname{codim} X-\operatorname{dim} X+1$. If $X$ is a degenerate scheme and $M^{i}(X) \cong M^{i}\left(X^{\prime}\right)\left(t_{0}\right)$ for some $t_{0} \in \mathbb{Z}, i=1, \ldots, k$, then $n=n^{\prime}, t_{0}=0, X^{\prime}$ is degenerate and $\left\langle X^{\prime}\right\rangle=\langle X\rangle$.

In particular, if $X$ is degenerate and $X^{\prime}$ is evenly G-linked to $X$, then $n=n^{\prime}$, $X^{\prime}$ is degenerate and $\left\langle X^{\prime}\right\rangle=\langle X\rangle$.

Proof. Note that the assumption $n>N-2 k+1$ ensures $n>2$ because the linear span of $X$ has dimension $\geq \operatorname{dim}\left(\left\langle Y_{1} \cup Y_{2}\right\rangle\right) \geq k+k+1$, and $\langle X\rangle \subset \mathbb{P}^{N}$, so $N \geq 2 k+1$ and $n>N-2 k+1 \geq 2$.

We first prove the assertion $n=n^{\prime}$. Since there exists $t_{0} \in \mathbb{Z}$ such that $M^{i}(X) \cong$ $M^{i}\left(X^{\prime}\right)\left(t_{0}\right)$ for $i=1, \ldots, k$, in particular we have $M^{1}(X) \cong M^{1}\left(X^{\prime}\right)\left(t_{0}\right)$. But since $X$ and $X^{\prime}$ are reduced schemes, it follows that $M^{1}(X)_{t}=M^{1}\left(X^{\prime}\right)_{t}=0$ for all $t<0$ and $M^{1}(X)_{0} \cong K^{n-1}$ and $M^{1}\left(X^{\prime}\right)_{0} \cong K^{n^{\prime}-1}$ (see [12, Theorem 1.2.6 (b)). So $t_{0}=0$ and $n=n^{\prime}$.

Now we will prove that the hyperplanes containing $X$ and the hyperplanes containing $X^{\prime}$ coincide. Indeed, if $H_{L}$ is a hyperplane containing $X, L \in R_{1}$, we have proved in Lemma 3.3 that $\phi_{t}^{X}(L)=0$ for all $t \in \mathbb{Z}$; so $L^{*} \in V_{0,0}(X)$. Furthermore, since $X$ satisfies the hypothesis of Lemma 3.4, we deduce from this lemma that

$$
V_{0,0}(X)=\left\{L^{*} \in\left(\mathbb{P}^{N}\right)^{*} \mid H_{L} \supset X\right\} .
$$

Analogously, since $n=n^{\prime}, X^{\prime}$ also satisfies the hypothesis of Lemmas [3.3] and [3.4] and we have

$$
V_{0,0}\left(X^{\prime}\right)=\left\{L^{*} \in\left(\mathbb{P}^{N}\right)^{*} \mid H_{L} \supset X^{\prime}\right\}
$$

We know that the loci $V_{0,0}$ are isomorphism invariants. Therefore $V_{0,0}(X)=$ $V_{0,0}\left(X^{\prime}\right)$ or, equivalently,

$$
\left\{L^{*} \in\left(\mathbb{P}^{N}\right)^{*} \mid H_{L} \supset X\right\}=\left\{L^{*} \in\left(\mathbb{P}^{N}\right)^{*} \mid H_{L} \supset X^{\prime}\right\} .
$$

Thus, $\langle X\rangle=\left\langle X^{\prime}\right\rangle$, which proves what we want.

The last assertion of the proposition follows directly from the HartshorneSchenzel Theorem 2.2 
Remarks 3.7. (1) In [11, Remark 2.5, J. Migliore conjectured that "if $C$ is any degenerate, not arithmetically Cohen-Macaulay curve in $\mathbb{P}^{4}$ and $C^{\prime}$ is in the same CI-liaison class of $C$ in $\mathbb{P}^{4}$, then they lie in the same hyperplane and are CI-linked inside that hyperplane". The same statement in the G-liaison context is false in general (see [4] and 9] for examples of arithmetically Buchsbaum curves that do not satisfy this conjecture). However, due to Proposition 3.6 and the Rao Theorem for curves in $\mathbb{P}^{3}$, we have that the conjecture holds if $C$ and $C^{\prime} \subset \mathbb{P}^{4}$ are evenly linked reduced curves having more than 3 connected components (notice that by [2], Theorem 2.3, $C$ and $C^{\prime}$ are not arithmetically Buchsbaum curves in this case).

(2) Once more, in Proposition 3.6 the assumption $n>N-2 k+1$ cannot be avoided. Indeed, if we consider $X \subset \mathbb{P}^{4}$ to be the disjoint union of two lines, then $X$ is a degenerate arithmetically Buchsbaum scheme; but we know by 4 . (or also 9]) that $X$ is G-linked to any other union of two skew lines in $\mathbb{P}^{4}$ (not necessarily lying in the same $\left.\mathbb{P}^{3}\right)$. If we change the assumption $n>N-2 k+1$ to $n>N-2 k+1-\varepsilon_{X}$ as in Remark 3.5, then we can guarantee only that $\langle X\rangle \subset\left\langle X^{\prime}\right\rangle$, but not the equality $\langle X\rangle=\left\langle X^{\prime}\right\rangle$. In order to have $\langle X\rangle=\left\langle X^{\prime}\right\rangle$ we need to verify also that $n^{\prime}=n>N-2 k+1-\varepsilon_{X^{\prime}}$. For instance, if $X$ and $X^{\prime}$ are unions of 3 degenerate skew lines in $\mathbb{P}^{4}, X \subset H, X^{\prime} \subset H^{\prime}$, and we assume that $X$ and $X^{\prime}$ are evenly G-linked, $X \sim X^{\prime}$, then we can ensure that $\langle X\rangle=\left\langle X^{\prime}\right\rangle$, because $\varepsilon_{X}=\varepsilon_{X^{\prime}}$ and $3>N-2 k+1-\varepsilon_{X}=N-2 k+1-\varepsilon_{X^{\prime}}=2$. But if we consider $Y$ to be the non-degenerate union of 3 skew lines in $\mathbb{P}^{4}$, and we assume that $X$ and $Y$ are evenly G-linked, then we can only say that $\langle X\rangle=H \subset\langle Y\rangle=\mathbb{P}^{4}$, which is obvious.

\section{UNIQUENESS OF VARIETIES OF MINIMAL DEGREE CONTAINING A GIVEN SCHEME}

It is known that any non-degenerate irreducible variety $X \subset \mathbb{P}^{N}$ satisfies $\operatorname{deg} X \geq$ $\operatorname{codim} X+1$, and the varieties that attain this bound are called varieties of minimal degree. First of all we will be interested in one type of variety of minimal degree: rational normal scrolls. Concerning rational normal scrolls, we use the same notation as [6]: $S\left(a_{0}, \ldots, a_{k}\right) \subset \mathbb{P}^{N}$ is a rational normal scroll of dimension $k+1$ and degree $c:=\sum a_{i}$ in $\mathbb{P}^{N}, N \geq c+k$. A rational normal scroll $S=S\left(a_{0}, \ldots, a_{k}\right)$ is smooth if and only if $a_{0}>0$ or $S=S(0, \ldots, 0,1)=\mathbb{P}^{k+1}$. Since we are not interested in divisors on $\mathbb{P}^{N}$, we will always exclude the case $S=\mathbb{P}^{k+1}$, so when we refer to $S$ smooth we will always mean $a_{0}>0$. The divisor class group of a rational normal scroll $S=S\left(a_{0}, \ldots, a_{k}\right) \subset \mathbb{P}^{c+k}, k \geq 1$, is generated by the hyperplane section $H$ and a linear subspace $F \subset S$ of dimension $k$. The canonical class of $S$ is $K_{S} \sim-(k+1) H+(c-2) F$.

We will now apply the results of the previous section to divisors of rational normal scrolls. Since our schemes are assumed to be locally Cohen-Macaulay and we are not interested in studying arithmetically Cohen-Macaulay schemes, the rational normal scrolls we consider in this subsection will all be smooth:

Remark 4.1. If $X$ is a locally Cohen-Macaulay scheme but not an arithmetically Cohen-Macaualy scheme, then $X$ cannot be contained on any singular rational normal scroll (see [3], Remark 3.1.12).

We will use the following known result (see [3], Lemmas 3.1.11 and 3.3.3, or [14]) that allows us to quickly identify $r$-Buchsbaum divisors on a smooth rational normal scroll. 
Lemma 4.2. Let $X \sim a H+b F$ be an effective divisor on a smooth rational normal scroll $S=S\left(a_{0}, \ldots, a_{k}\right) \subset \mathbb{P}^{N}$. Then:

i) $X$ is 0 -Buchsbaum (or $A C M$ ) if and only if $1-c \leq b \leq 1$.

ii) If $b>1$, then $X$ is an $r$-Buchsbaum scheme if and only if $b=(r-1) a_{0}+p+2$ for some $0 \leq p<a_{0}$ (in other words, $r=\left\lfloor\frac{b-2}{a_{0}}\right\rfloor+1$ ). In this case, $M^{i}(X)=0$ for $i=2, \ldots, k ; M^{1}(X)_{l} \neq 0$ if and only if $a \leq l \leq\left\lfloor\frac{b-2}{a_{0}}\right\rfloor+a$, and $\operatorname{diam} M^{1}(X)=r$.

iii) If $b<1-c$, then $X$ is an $r$-Buchsbaum scheme if and only if $-c+2-b=$ $(r-1) a_{0}+p+2$ for some $0 \leq p<a_{0}$. In this case, $M^{i}(X)=0$ for $i=1, \ldots, k-1 ; M^{k}(X)_{l} \neq 0$ if and only if $\left\lfloor\frac{-c-b}{a_{0}}\right\rfloor+a-k-1 \leq l \leq a-k-1$, and $\operatorname{diam} M^{k}(X)=r$.

To begin with the results of this section, we will give a way to decide whether the general hyperplane section of a disjoint union of linear varieties of dimension $k, X=\bigcup_{i=1}^{n} L_{i} \subset \mathbb{P}^{N}$, lying on a smooth rational normal scroll $S\left(a_{0}, \ldots, a_{k}\right)$ is degenerate or not. To this end, we introduce some notation:

Definition 4.3 (1], Definition 2.1). Let $S=S\left(a_{0}, \ldots, a_{k}\right) \subset \mathbb{P}^{c+k}$ be a rational normal scroll. We define the index of relative balance $r(S)$ of $S$ as

$$
r(S):=k+1-\max \left\{j \mid j a_{j} \leq \sum_{i=0}^{j} a_{j}\right\} .
$$

Note that $r(S) \leq k$ because $a_{1} \leq a_{0}+a_{1}$.

Let $S=S\left(a_{0}, \ldots, a_{k}\right) \subset \mathbb{P}^{c+k}$ be a rational normal scroll and let $H$ be a hyperplane such that $S^{\prime}:=H \cap S$ is again a rational normal scroll (this is the case if $H$ does not contain any fiber of $S)$. Write $S^{\prime}=S\left(a_{0}^{\prime}, \ldots a_{k-1}^{\prime}\right)$; then, according to [1], Theorem 2.4,

$$
\begin{aligned}
& \text { 1. } \sum_{i=0}^{k-1} a_{i}^{\prime}=c \text {, } \\
& \text { 2. } a_{k-r(S)}^{\prime} \leq a_{0}^{\prime}+1 \text {, and } \\
& \text { 3. } a_{k-i}^{\prime}=a_{k+1-i} \quad 1 \leq i \leq r(S)-1 .
\end{aligned}
$$

In particular, we deduce from 1,2 and 3 that if $r(S) \geq 2$ (or equivalently $a_{k}>\frac{c}{k}$ ), then $a_{k-1}^{\prime}=a_{k}$; and if $r(S)=1$ (i.e. $c \geq k a_{k}$ ), then $a_{0}^{\prime}=\left\lfloor\frac{c}{k}\right\rfloor$ and $a_{i}^{\prime} \leq\left\lfloor\frac{c}{k}\right\rfloor+1$ for $i=1, \ldots, k-1$. We have

Lemma 4.4. Let $S=S\left(a_{0}, \ldots, a_{k}\right) \subset \mathbb{P}^{c+k}, c=\sum_{i=0}^{k} a_{i}$, be a smooth rational normal scroll. An effective divisor $Y \sim b F$ on $S$ is non-degenerate if and only if $b \geq a_{k}+1$.

Proof. Since $S$ is non-degenerate and ACM, the exact sequence

$$
0 \rightarrow \mathcal{I}_{S} \rightarrow \mathcal{I}_{Y} \rightarrow \mathcal{I}_{Y, S} \cong \mathcal{O}_{S}(-Y) \rightarrow 0
$$

gives an exact sequence on cohomology

$$
0=H^{0}\left(\mathcal{I}_{S}(1)\right) \longrightarrow H^{0}\left(\mathcal{I}_{Y}(1)\right) \longrightarrow H^{0}\left(\mathcal{O}_{S}(H-Y)\right) \longrightarrow H^{1}\left(\mathcal{I}_{S}(1)\right)=0 .
$$

Hence, $h^{0}\left(\mathcal{I}_{Y}(1)\right)=h^{0}\left(\mathcal{O}_{S}(H-b F)\right)=\sum_{i=0}^{k} \max \left(a_{i}-b+1,0\right)$ (see [6] or [3] for results on the cohomology of divisors on rational normal scrolls). So, $h^{0}\left(\mathcal{I}_{Y}(1)\right)=0$ if and only if $a_{i}-b+1 \leq 0$ for all $i=0, \ldots, k$, i.e., $b \geq a_{k}+1$. 
Corollary 4.5. Let $X \sim b F$ be a non-degenerate divisor of a smooth rational normal scroll $S=S\left(a_{0}, \ldots, a_{k}\right) \subset \mathbb{P}^{c+k}$. Assume $b \geq\left\lceil\frac{c}{k}\right\rceil+1$. If $H$ is a hyperplane not containing any fiber of $S$, then $X \cap H \subset S^{\prime}=S\left(a_{0}^{\prime}, \ldots, a_{k-1}^{\prime}\right) \subset H \cong \mathbb{P}^{c+k-1}$ is also non-degenerate.

Proof. First of all, note that since $X$ is non-degenerate, applying Lemma 4.4 we get $b \geq a_{k}+1$. We now distinguish two cases depending on the index of relative balance $r(S)$ of $S$ :

Case (1): $r(S) \geq 2$. In this case, $b \geq a_{k}+1=a_{k-1}^{\prime}+1$, and Lemma 4.4 proves that $X \cap H$ is non-degenerate.

Case (2): $r(S)=1$. Then $a_{0}^{\prime}=\left\lfloor\frac{c}{k}\right\rfloor$ (use 1, 2 and 3 on the previous page) and $a_{k-1}^{\prime} \leq\left\lfloor\frac{c}{k}\right\rfloor+1$. If in addition $\frac{c}{k}$ is not an integer, then $b \geq\left\lceil\frac{c}{k}\right\rceil+1>\left\lfloor\frac{c}{k}\right\rfloor+2 \geq$ $a_{k-1}^{\prime}+1$, and $X \cap H$ is non-degenerate by the argument above. If $\frac{c}{k}$ is an integer, $c=\lambda k$, then $a_{0}^{\prime}=\lambda, a_{k-1}^{\prime} \leq \lambda+1$, and since $c=\sum_{i=0}^{k-1} a_{i}^{\prime}$, we obtain in fact that $a_{k-1}^{\prime}=\lambda=\left\lceil\frac{c}{k}\right\rceil$. Thus, $b \geq\left\lceil\frac{c}{k}\right\rceil+1=a_{k-1}^{\prime}+1$, and Lemma 4.4 gives again that $X \cap H$ is non-degenerate.

We are now ready to state and prove the main results of this section. In what follows, the rational normal scrolls we consider are non-degenerate because the degenerate case can be avoided by Proposition 3.6

Proposition 4.6. Let $X=\bigcup_{i=1}^{n} L_{i} \subset \mathbb{P}^{N}$ and $X^{\prime}=\bigcup_{i=1}^{n^{\prime}} L_{i}^{\prime} \subset \mathbb{P}^{N}$ be disjoint unions of linear varieties of dimension $k \geq 1$. Assume that $X$ is a union of fibers of a smooth rational normal scroll $S=S\left(a_{0}, \ldots, a_{k}\right) \subset \mathbb{P}^{N}, N=c+k$, and that $n>c-k+1$.

Then $X$ and $X^{\prime}$ have isomorphic Rao module (up to shift) if and only if $n=n^{\prime}$ and $X^{\prime}$ is also a divisor on $S$.

Remark 4.7. Under the hypothesis of this proposition, $X$ cannot be an arithmetically Buchsbaum scheme in any case. Indeed, since $X$ is linearly equivalent to $n F$ in $S$ and $n>c-k+1 \geq a_{0}+k-k+1$ (because $S$ is smooth), we have that $n \geq a_{0}+2$, and by Lemma 4.2 we obtain that $X$ is $r$-Buchsbaum with $r \geq 2$. Therefore, Proposition 4.6 does not contradict the results of [4].

Proof of Proposition 4.6. Note first that since $S$ is smooth and we have assumed $n>c-k+1$, we have $n>a_{k}+k-k+1=a_{k}+1$. Thus, by Lemma 4.4, $X$ is non-degenerate.

We observe now that for any hyperplane $H$ not containing any fiber of $S, X \cap H \subset$ $H$ is non-degenerate. Indeed, the assumption $n \geq c-k+2$ implies $n \geq\left\lceil\frac{c}{k}\right]+1$, because $c-k \geq \frac{c}{k}-1$ if and only if $c \frac{k-1}{k} \geq k-1$, which is true because $c \geq k+1$. Hence, by Corollary $4.5, X \cap H$ is non-degenerate.

Suppose that $n=n^{\prime}$ and $X^{\prime}$ also lies on the rational normal scroll $S$. Then $X^{\prime}$ is also linearly equivalent to $n F$, so $X$ and $X^{\prime}$ are evenly G-linked thanks to [8], Corollary 5.13. In particular, by the Hartshorne-Schenzel Theorem [2.2, $X$ and $X^{\prime}$ have isomorphic Rao module up to some shift.

We are going to prove the converse. Assume that $X$ and $X^{\prime}$ have isomorphic Rao module, $M^{1}(X) \cong M^{1}\left(X^{\prime}\right)(t)$ for some $t \in \mathbb{Z}$. Since both $M^{1}(X)$ and $M^{1}\left(X^{\prime}\right)$ start in degree 0 , we have $t=0$; and since $M^{1}(X) \cong K^{n-1}, M^{1}\left(X^{\prime}\right) \cong K^{n^{\prime}-1}$, we obtain $n=n^{\prime}$. 
Notice that the general hyperplane section of $X^{\prime}$ is non-degenerate. Indeed, let $H_{L}$ be a general hyperplane. If we consider the exact sequence of sheaves

$$
\begin{gathered}
0 \longrightarrow \mathcal{I}_{X} \longrightarrow \mathcal{I}_{X}(1) \longrightarrow \mathcal{I}_{X \cap H, H}(1) \longrightarrow 0 \\
\text { (resp. } \left.0 \longrightarrow \mathcal{I}_{X^{\prime}} \longrightarrow \mathcal{I}_{X^{\prime}}(1) \longrightarrow \mathcal{I}_{X^{\prime} \cap H, H}(1) \longrightarrow 0\right)
\end{gathered}
$$

and we take cohomology, we get the exact sequence

$$
\begin{gathered}
0 \longrightarrow H^{0}\left(\mathbb{P}^{N}, \mathcal{I}_{X \cap H, H}(1)\right) \longrightarrow M^{1}(X)_{0} \stackrel{\phi_{0}(L)}{\longrightarrow} M^{1}(X)_{1} \\
\text { (resp. } \left.0 \longrightarrow H^{0}\left(\mathbb{P}^{N}, \mathcal{I}_{X^{\prime} \cap H, H}(1)\right) \longrightarrow M^{1}\left(X^{\prime}\right)_{0} \stackrel{\phi_{0}^{\prime}(L)}{\longrightarrow} M^{1}\left(X^{\prime}\right)_{1}\right) .
\end{gathered}
$$

As we have proved that the general hyperplane section of $X$ is non-degenerate, we obtain that $\operatorname{rk} \phi_{0}(L)=n-1$ for a general $H_{L}$. Now, since $M^{1}(X)$ and $M^{1}\left(X^{\prime}\right)$ are isomorphic as graded $R$-modules, we also have that $\operatorname{rk} \phi_{0}^{\prime}(L)=n-1$ for a general $L$, and hence the general hyperplane section $X^{\prime} \cap H$ is non-degenerate.

Since we have proved that $X$ (resp. $X^{\prime}$ ) and its general hyperplane section (resp. the general hyperplane section of $X^{\prime}$ ) are non-degenerate, we have verified that $X$ and $X^{\prime}$ satisfy the hypothesis of Lemma 3.2. We define

$$
\begin{aligned}
X^{*} & :=\left\{L^{*} \in\left(\mathbb{P}^{N}\right)^{*} \mid H_{L} \text { contains an irreducible component of } X\right\} \subset\left(\mathbb{P}^{N}\right)^{*}, \\
X^{\prime *} & :=\left\{L^{*} \in\left(\mathbb{P}^{N}\right)^{*} \mid H_{L} \text { contains an irreducible component of } X^{\prime}\right\} \subset\left(\mathbb{P}^{N}\right)^{*} .
\end{aligned}
$$

Applying Lemma 3.2, we get that $X^{*} \subset V_{0}(X)$ and $X^{\prime *} \subset V_{0}\left(X^{\prime}\right)$.

Let us define $A:=\left\{L^{*} \mid H_{L}\right.$ contains some fiber of $\left.\mathrm{S}\right\} \subset\left(\mathbb{P}^{N}\right)^{*}$. We are going to check that $V_{0}(X) \subseteq A$. Indeed, we consider $L^{*}$ an element of $V_{0}(X)$. If $L^{*}$ does not belong to $U_{X}$, then $H_{L}$ contains some $L_{i}$ which is a fiber of $S$; so $L^{*} \in A$. If $L^{*}$ belongs to $U_{X}$ and $H_{L}$ does not contain any fiber of $S$, then $h^{0}\left(H_{L}, \mathcal{I}_{X \cap H_{L}}(1)\right)=0$, as we have proved above. Therefore, by Lemma 3.1, $V_{0}(X) \subseteq A$.

On the other hand, since $V_{0}(X)$ are isomorphism invariants of the first deficiency modules, we have

$$
X^{\prime *} \subseteq V_{0}\left(X^{\prime}\right)=V_{0}(X) \subseteq A .
$$

But from these inclusions we deduce that for any hyperplane $H_{L}$ containing one of the $L_{i}^{\prime}$ (i.e. $\left.L^{*} \in X^{\prime *}\right), H_{L}$ contains some fiber of $S$. But this is not true unless $L_{i}^{\prime}$ are also fibers of $S$. So $X^{\prime} \subset S$, as we wanted to prove.

The next corollary, generalizing [10], Theorem 3.1, immediately follows from the Hartshorne-Schenzel Theorem 2.2 and Proposition 4.6

Corollary 4.8. Let $X=\bigcup_{i=1}^{n} L_{i} \subset \mathbb{P}^{N}$ and $X^{\prime}=\bigcup_{i=1}^{n^{\prime}} L_{i}^{\prime} \subset \mathbb{P}^{N}$ be disjoint unions of linear varieties of dimension $k \geq 1$. Assume that $X$ is a union of fibers of a smooth rational normal scroll $S=S\left(a_{0}, \ldots, a_{k}\right) \subset \mathbb{P}^{c+k}, N=c+k$, and that $n>c-k+1$. If $X^{\prime}$ is evenly $G$-linked to $X$, then $X^{\prime} \subset S$.

Proposition 4.6 allows us to prove the main result of this paper:

Theorem 4.9. Let $X \subset \mathbb{P}^{N}$ be a non-degenerate subscheme of dimension $k \geq 1$ that is a divisor on a smooth rational normal scroll $S \subset \mathbb{P}^{N}$ of degree $c$ (so $N=c+k$ ). We write $X \sim a H+b F \subset S$ and we assume that either $b>c-k+1$ or $b<k+1-2 c$. Then $S$ is the only rational normal scroll of dimension $k+1$ containing $X$. 
Proof. Assume that there is another rational normal scroll $S^{\prime}=S\left(a_{0}^{\prime}, \ldots, a_{k}^{\prime}\right) \subset \mathbb{P}^{N}$ containing $X$. As $X$ is non-degenerate, $S^{\prime}$ is also non-degenerate, so $\operatorname{deg} S^{\prime}=c$.

1st case: $b>c-k+1$.

Claim: In $S^{\prime}, X$ is linearly equivalent to $a H+b F$.

Proof of claim. If $X$ is linearly equivalent to $\alpha H+\beta F$ on $S^{\prime}$, for some integers $\alpha, \beta$, then looking at the deficiency modules and using Lemma 4.2 we see that there are two possibilities:

1) $\beta>1$. In this case, again by Lemma 4.2 we see that $a=\alpha$ because $a$ (resp. $\alpha$ ) is the first degree for which the Rao module of $X$ is non-zero. Hence we have $\operatorname{deg} X=a c+b=a c+\beta$, and so get $b=\beta$, and we are done.

2) $k=1$ and $\beta<1-c$. We will see that this case cannot occur. Indeed, in this case, looking at the first and last degree in which the Rao module of $X$ is non-zero, by Lemma 4.2, we have $a=\alpha-2+\left\lfloor\frac{-c-\beta}{a_{0}^{\prime}}\right\rfloor$ and $\left\lfloor\frac{b-2}{a_{0}}\right\rfloor+a=\alpha-2$. Thus, $-\left\lfloor\frac{-c-\beta}{a_{0}^{\prime}}\right\rfloor=\left\lfloor\frac{b-2}{a_{0}}\right\rfloor$; but the left term in this equality is $\leq 0$ and the right term is $\geq 0$, so $\left\lfloor\frac{b-2}{a_{0}}\right\rfloor=0$. But we were assuming that $b>c-k+1=c$, so $b-2 \geq c-1 \geq 2 a_{0}-1 \geq a_{0}$, and hence $\left\lfloor\frac{b-2}{a_{0}}\right\rfloor$ cannot be 0 (in other words, $X$ cannot be 1-Buchsbaum). The claim is proved.

Since $b>c-k+1$, by 8 , Corollary $5.13, X$ is evenly G-linked to the union $Y=$ $\bigcup_{i=1}^{b} L_{i}$ (resp. $Y^{\prime}=\bigcup_{i=1}^{b} L_{i}^{\prime}$ ) of $b$ different fibers of $S$ and to a union $Y^{\prime}=\bigcup_{i=1}^{b} L_{i}^{\prime}$ of $b$ different fibers of $S^{\prime}$. Thus $Y$ and $Y^{\prime}$ have isomorphic deficiency modules up to shift (due to the Hartshorne-Schenzel Theorem), and we obtain by Proposition 4.6 that $Y \subset S^{\prime}$ and $Y^{\prime} \subset S$. Furthermore, this argument can be done for any set of $b$ fibers of $S$ (since any set of $b$ fibers of $S$ has deficiency modules isomorphic to $X$ and we can apply Proposition 4.6), so that any set of $b$ different fibers of $S$ is in $S^{\prime}$. This directly implies $S=S^{\prime}$, as we wanted to prove.

2nd case: $b<k+1-2 c$.

If $b<k+1-2 c$, then using [8], Corollary 5.5, we perform a G-link $X^{m H_{-K S}} Z$

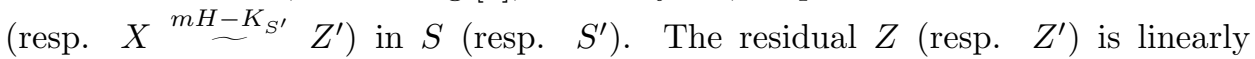
equivalent in $S$ (resp. $\left.S^{\prime}\right)$ to $(m-a+k+1) H+(-c+2-b) F$. As $b<k+1-2 c$, we obtain $-c+2-b>c-k+1$ and by [8, Corollary 5.14, we have that $Z$ (resp. $Z^{\prime}$ ) is evenly G-linked to a set $Y$ (resp. $Y^{\prime}$ ) of $-c+2-b$ fibers of $S$ (resp. $S^{\prime}$ ). Using with $Y$ and $Y^{\prime}$ the same arguments as in the previous paragraph, we conclude that $S=S^{\prime}$.

As a consequence of the classification of varieties of minimal degree we also get

Corollary 4.10. If $X \subset \mathbb{P}^{N}$ is a non-degenerate $r$-Buchsbaum scheme of dimension $k \geq 1$ and codimension $c$ with $r>\max (c-k, 0)=\max (N-2 k, 0)$, then $X$ is contained in at most one variety of minimal degree of dimension $k+1$.

Proof. The theorem classifying varieties of minimal degree (see [5], [6]) says that if $V \subset \mathbb{P}^{N}$ is a non-degenerate irreducible variety of minimal degree, then $V$ is either a rational normal scroll, or a cone over the Veronese surface $\mathbb{P}^{2} \hookrightarrow \mathbb{P}^{5}$, or the Veronese surface itself, or a hyperquadric of rank greater than 4 .

Since any divisor on a hyperquadric of rank greater than 4, or on a cone over the Veronese surface in $\mathbb{P}^{5}$, or on the Veronese surface in $\mathbb{P}^{5}$ is an arithmetically CohenMacaulay scheme and $X$ is assumed to be $r$-Buchsbaum with $r>0$, we conclude that $X$ cannot be a divisor on any of these varieties. Hence, $X$ is contained on a 
rational normal scroll $S=S\left(a_{0}, \ldots, a_{k}\right)$. Moreover, as $X$ is locally Cohen-Macaulay but not arithmetically Cohen-Macaulay, the rational normal scroll $S$ containing it is smooth (see Remark 4.1), so $a_{0} \geq 1$ and $c \geq k+a_{0} \geq k+1$. Assume that $X$ is linearly equivalent to $a H+b F$ on $S$. We are now going to check that $X$ satisfies the hypothesis of Theorem 4.9 .

As $X$ is $r$-Buchsbaum, by Lemma 4.2 we have $b=(r-1) a_{0}+p+2$ or $b=$ $-c+2-(r-1) a_{0}-p-2$ for some $0 \leq p<a_{0}$.

Since we are assuming $r>c-k$ and we have seen that $a_{0} \geq 1$ and $c-k \geq 1$, in the first case we have $b \geq(c-k) a_{0}+p+2 \geq(c-k) a_{0}+2>c-k+1$ and in the second case we have $b \leq-c+2-(c-k) a_{0}-p-2 \leq-2 c+k<-2 c+k+1$. Therefore $X$ satisfies the hypothesis of Theorem 4.9, and we conclude that $S$ is the unique rational normal scroll containing $X$. Summing up, we have that $X$ is contained on a unique variety of minimal degree.

Remarks 4.11. (1) As we have seen in the proof of Corollary 4.10, if $X$ is $r$ Buchsbaum with $r>\max (c-k, 0)$ and $X$ lies on a variety of minimal degree, then $c-k \geq 1$. Hence $X$ cannot be 1-Buchsbaum. In fact, when $X$ is arithmetically Buchsbaum, then $X$ can be contained in more than one smooth rational normal scroll (see [4]).

(2) The bound $r>c-k$ cannot be improved in general. Indeed, if we consider curves in $\mathbb{P}^{3}$ (so $c=2$ and $k=1$ ), then if $X$ is the union of two skew lines, it follows that $X$ is 1 -Buchsbaum $(1=c-k)$ and $X$ is contained in more than one smooth quadric surface in $\mathbb{P}^{3}$. If we consider curves in $\mathbb{P}^{4}$, the case of three skew lines is the only case of non-arithmetically Buchsbaum curves not covered by Theorem 4.9 or Corollary 4.10. But if $X \subset \mathbb{P}^{4}$ is the disjoint union of 3 general skew lines, then $X$ is contained in more than one rational normal scroll $S(1,2) \subset \mathbb{P}^{4}$. We can construct these rational normal scrolls as follows: $X$ has a unique trisecant line $Z$, and we consider a plane $\pi \subset \mathbb{P}^{4}$ such that $\pi \cap Z=\emptyset$. In this plane $\pi$ we can find infinitely many conics through $X \cap \pi$ such that the rational normal scroll generated by $Z$ and the conic contains $X$.

The following corollary clearly generalizes [10, Theorem 3.2:

Corollary 4.12. Let $X, X^{\prime} \subset \mathbb{P}^{N}$ be two subschemes of dimension $k \geq 1, N=c+k$. Suppose that $X \subset S \subset \mathbb{P}^{N}$ (resp. $X^{\prime} \subset S^{\prime} \subset \mathbb{P}^{N}$ ) is a divisor of a rational normal scroll $S$ (resp. $S^{\prime}$ ) of degree c. We write $X \sim a H+b F \subset S$ and $X^{\prime} \sim a^{\prime} H+b^{\prime} F \subset$ $S^{\prime}$, and we assume that either $b, b^{\prime}>c-k+1$ or $b, b^{\prime}<k+1-2 c$. Then the following conditions are equivalent:

(i) $X$ and $X^{\prime}$ are evenly G-linked.

(ii) The deficiency modules of $X$ and $X^{\prime}$ are isomorphic (up to shift).

(iii) $b=b^{\prime}$ and $S=S^{\prime}$.

Proof. $(i) \Rightarrow(i i)$. If $X$ and $X^{\prime}$ are evenly G-linked, then their deficiency modules are isomorphic (up to shift) by the Hartshorne-Schenzel Theorem (see [12], Theorem 5.3.1).

(ii) $\Rightarrow$ (iii). If $b, b^{\prime}>c-k+1$, then by [8, Corollary 5.14, $X$ (and resp. $X^{\prime}$ ) is evenly G-linked to the union $Y=\bigcup_{i=1}^{b} L_{i}$ (resp. $\left.Y^{\prime}=\bigcup_{i=1}^{b^{\prime}} L_{i}^{\prime}\right)$ of $b$ different fibers of $S$ (resp. $b^{\prime}$ different fibers of $S^{\prime}$ ). Since we are assuming that $X$ and $X^{\prime}$ have isomorphic deficiency modules (up to shift), it follows that $Y$ and $Y^{\prime}$ also have isomorphic deficiency modules (up to shift) by the Hartshorne-Schenzel Theorem. 
Now by Proposition 4.6 we get $b=b^{\prime}, Y \subset S^{\prime}$ (and $Y^{\prime} \subset S$ ). Thus Theorem 4.9 gives us that $S=S^{\prime}$, as we wanted to prove.

If $b, b^{\prime}<k+1-2 c$, then, using [8], Corollary 5.5, again we perform a G-link $X \stackrel{m H-K_{S}}{\sim} Y$ in $S$ (resp. $X^{\prime}{ }^{m^{\prime} H-K_{S^{\prime}}} Y^{\prime}$ in $S^{\prime}$ ). The residual $Y\left(\right.$ resp. $Y^{\prime}$ ) is linearly equivalent in $S$ (resp. in $\left.S^{\prime}\right)$ to $(m-a+k+1) H+(-c+2-b) F$ (resp. to $\left.\left(m^{\prime}-a^{\prime}+k+1\right) H+\left(-c+2-b^{\prime}\right) F\right)$. Since $X$ and $X^{\prime}$ have isomorphic deficiency modules (up to shift), so do $Y$ and $Y^{\prime}$. As $b, b^{\prime}<k+1-2 c$, we obtain $-c+2-b>c-k+1$ and $-c+2-b^{\prime}>c-k+1$, and using the first case considered above applied to $Y$ and $Y^{\prime}$, we conclude that $S=S^{\prime}$ and $-c+2-b=-c+2-b^{\prime}$ (so $b=b^{\prime}$ ).

(iii) $\Rightarrow(i)$. If $b=b^{\prime}$ and $S=S^{\prime}$, then we conclude by [8], Corollary 5.14, that $X$ and $X^{\prime}$ are evenly G-linked.

Examples 4.13. We give here examples of G-liaison classes of schemes that satisfy the hypotheses of Corollary 4.12

1) Let $X \subset \mathbb{P}^{4}$ (resp. $\left.X^{\prime} \subset \mathbb{P}^{4}\right)$ be a non-degenerate union of 4 skew lines that have a 4 -secant line $L$ (resp. $L^{\prime}$ ) and such that there is a hyperplane $H \nsupseteq L$ (resp. $\left.H^{\prime} \nsupseteq L^{\prime}\right)$ meeting $X$ (resp. $\left.X^{\prime}\right)$ on a degenerate set of points. Then $X\left(\right.$ resp. $\left.X^{\prime}\right)$ lies on a unique rational normal scroll $S=S(1,2) \subset \mathbb{P}^{4}\left(\right.$ resp. $\left.S^{\prime}=S(1,2) \subset \mathbb{P}^{4}\right)$ (we can construct it as in Remark $4.11(2)$ ). Therefore, according to Corollary 4.12 $X$ and $X^{\prime}$ belong to the same even G-liaison class if and only if $S=S^{\prime}$. In this case, $X$ and $X^{\prime}$ share the same common 4 -secant line.

2) Let $C, C^{\prime} \subset \mathbb{P}^{N}$ be two smooth, non-degenerate, linearly normal, hyperelliptic irreducible curves. If $C, C^{\prime}$ are $r$-Buchsbaum with $r>1$ and $\operatorname{deg} C$, $\operatorname{deg} C^{\prime}>3 N-3$, then $C$ (resp. $C^{\prime}$ ) is a divisor on a rational normal scroll $S \subset \mathbb{P}^{N}$ (resp. $S^{\prime} \subset \mathbb{P}^{N}$ ) and $C, C^{\prime}$ are evenly G-linked if and only if $S=S^{\prime}$ and $\operatorname{deg} C=\operatorname{deg} C^{\prime}$. Indeed, in this case there exists a linear system of dimension 1 and degree 2 on $C$, and we denote by $D$ the divisor defining this linear system. Then we apply [5], Theorem 2 , to $C$ and $D$, and we get that there exists a rational normal scroll $S \subset \mathbb{P}^{N}$ of dimension $N+1-h^{0}\left(\mathcal{O}_{C}\left(H_{C}-D\right)\right)$ and degree $h^{0}\left(\mathcal{O}_{C}\left(H_{C}-D\right)\right)$. Since $H_{C}$ is very ample and $D$ has degree $2, h^{0}\left(\mathcal{O}_{C}\left(H_{C}-D\right)\right)=h^{0}\left(\mathcal{O}_{C}\left(H_{C}\right)\right)-2=N-1$. Thus, we have a rational normal scroll surface $S \subset \mathbb{P}^{N}$ of degree $c:=N-1$ containing $C$. Moreover, if we write $C \sim a H+b F$ on $S$, we have $\operatorname{deg} C=a(N-1)+b$ and $a=C \cdot F$. Since, by the construction of [5], Theorem 2, the fibers $F$ of $S$ are the linear spans of divisors linearly equivalent to $D$, which are divisors of degree 2 on $C$, we have $a=C \cdot F=2$. Therefore $b=\operatorname{deg} C-2 N+2$ and $C \sim 2 H+(\operatorname{deg} C-2 N+2) F$. The same argument can be applied to $C^{\prime} \subset \mathbb{P}^{N}$, and we find a rational normal scroll surface $S^{\prime} \subset \mathbb{P}^{N}$, containing $C^{\prime}$ and $C^{\prime} \sim 2 H+\left(\operatorname{deg} C^{\prime}-2 N+2\right) F$ on $S^{\prime}$. If $\operatorname{deg} C, \operatorname{deg} C^{\prime}>3 N-3$ then $b=\operatorname{deg} C-2 N+2>N-1=c$ (resp. $b^{\prime}=\operatorname{deg} C^{\prime}-2 N+2>c$ ), and the hypotheses of Corollary 4.12 are verified. Thus, $C$ and $C^{\prime}$ are evenly G-linked if and only if $b=b^{\prime}$, i.e., $\operatorname{deg} C=\operatorname{deg} C^{\prime}$.

\section{REFERENCES}

1. J.N. Brawner, Tetragonal curves, rational normal scrolls and K3 surfaces, Trans. Amer. Math. Soc. 349 (1997), 3075-3091. MR 97j:14056

2. M. Casanellas, Characterization of non-connected Buchsbaum curves in $\mathbb{P}^{n}$, Le Matematiche (Catania) LIV (1999), 187-195. MR 2001j:14063

3. - Teoria de liaison en codimensió arbitrària, Ph.D. thesis, Universitat de Barcelona, 2002. 
4. M. Casanellas and R.M. Miró-Roig, Gorenstein liaison and special linear configurations, Illinois Math. J. 46 (2002), 129-143. MR 2003i:14062

5. D. Eisenbud and J. Harris, On varieties of minimal degree (A centennial account), Algebraic Geomtery, Bowdoin 1985, Amer. Math. Soc. Symp. in Pure and App. Math. (S. Bloch, ed.), vol. 46, 1985, pp. 3-14. MR 89f:14042

6. J. Harris, A bound on the geometric genus of projective varieties, Ann. Scuola Norm. Sup. Pisa (4) 8 (1981), 35-68. MR 82h:14010

7. L.T. Hoa, R.M. Miró-Roig, and W. Vogel, On numerical invariants of locally Cohen-Macaulay schemes in $\mathbb{P}^{n}$, Hiroshima Math. J. 24 (1994), 299-316. MR 95m:14031

8. J. Kleppe, J. Migliore, R.M. Miró-Roig, U. Nagel, and C. Peterson, Gorenstein liaison, complete intersection liaison invariants and unobstructedness, Memoirs A.M.S. 732 (2001). MR 2002e: 14083

9. J. Lesperance, Gorenstein liaison of some curves in $\mathbb{P}^{4}$, Collectanea Mathematica 52 (2001), 219-230. MR 2003g:14065

10. J. Migliore, Geometric invariants for liaison of space curves, J. Alg. 99 (1986), 548-572. MR 87g:14031

11. Liaison of a union of skew lines in $\mathbb{P}^{4}$, Pacific J. of Math. 130 (1987), 153-170. MR

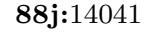

12. Introduction to liaison theory and deficiency modules, Progress in Mathematics, no, 165, Birkhäuser, 1998. MR 2000g:14058

13. J. Migliore and U. Nagel, Liaison and related topics, Rend. Sem. Mat. Univ. Pol. Torino 59 (2001), 59-126.

14. C. Miyazaki, Sharp bounds on Castelnuovo-Mumford regularity, Trans. Amer. Math. Soc. 352 (1999), 1675-1686. MR 2000i:13017

Departament d'Algebra i Geometria, Facultat de Matematiques, Universitat de Barcelona, Gran Via 585, 08007-Barcelona, Spain

E-mail address: casanell@mat.ub.es 